\title{
Telangiectasia nevoide unilateral adquirida
}

\section{Acquired unilateral nevoid telangiectasia}

\author{
Juan Ramón Trejo-Acuña, ${ }^{*}$ Maribet González-González, ${ }^{\ddagger}$ María José García-Alonso§
}

\section{RESUMEN}

La telangiectasia nevoide unilateral es un padecimiento poco frecuente, congénito 0 adquirido, que afecta principalmente a las mujeres, la cual se asocia a una influencia hormonal por hiperestrogenismo. Se caracteriza clínicamente por la presencia de múltiples telangiectasias distribuidas de forma lineal, unilateral, siguiendo las líneas de Blaschko. La telangiectasia nevoide unilateral suele ser una dermatosis persistente. No existe un tratamiento específico; sin embargo, en ocasiones, en los casos adquiridos, puede remitir de manera espontánea.

Palabras clave: Telangiectasia nevoide unilateral, hiperestrogenismo, líneas de Blaschko, hormonal.

\section{INTRODUCCIÓN}

La telangiectasia nevoide unilateral (TNU), también llamada Telangiectasia superficial del dermatoma unilateral, es un padecimiento poco frecuente, de incidencia desconocida. Fue descrita por primera vez en 1899 por Blaschko. ${ }^{1,2}$

Su fisiopatología exacta aún se desconoce, aunque algunos autores han detectado cierta asociación con estados de hiperestrogenismo, considerando la influencia de los receptores estrogénicos presentes en el endotelio vascular así como su papel en el proceso de angiogénesis. ${ }^{2,3}$

Se han publicado casos de TNU en pacientes durante la pubertad, el embarazo, por la administración de anticonceptivos orales, por la ingesta de terapia de sustitución hormonal, por alcoholismo,

\footnotetext{
* Dermatólogo.

‡ Dermatopatóloga.

$\S$ Residente del segundo año de Dermatología.

Centro Dermatológico «Dr. Ladislao de la Pascua», SSCDMX.
}

\section{ABSTRACT}

Unilateral nevoid telangiectasia is a rare condition that can have a congenital or acquired origin. It is more common in women, and can be associated with hormonal influence such as hyperestrogenism. Clinically it is characterized by multiple, unilateral, linearly arranged telangiectases in a Blaschko pattern. Unilateral nevoid telangiectasia is usually a persistent condition, and it has not a specific treatment, but sometimes, in acquired cases, it can remit spontaneously.

Keywords: Unilateral nevoid telangiectasia, hyperestrogenism, Blaschko pattern, hormonal. cirrosis, hipertensión portal, síndrome carcinoide e hipertiroidismo. ${ }^{2}$

Un hallazgo importante es que esta dermatosis se ha observado en pacientes hombres y mujeres previamente sanos, por lo que las asociaciones mencionadas no siempre están presentes. ${ }^{4}$

Las manifestaciones cutáneas son el resultado del mosaicismo de células mesodérmicas, lo cual condiciona su distribución siguiendo las líneas de Blaschko. ${ }^{5} \mathrm{Se}$ ha observado que ciertos factores estrogénicos desencadenan la proliferación y dilatación vascular, y que los niveles de receptores estrogénicos y de progesterona en la piel afectada de estos pacientes se encuentran incrementados, además se sugiere que pueden existir alteraciones en dichos receptores, lo cual favorece que los estrógenos circulantes desencadenen un proceso de angiogénesis. ${ }^{6}$

La TNU puede ser congénita o adquirida. La forma congénita es muy rara; tiene un patrón de herencia au-

Citar como: Trejo-Acuña JR, González-González M, García-Alonso MJ. Telangiectasia nevoide unilateral adquirida. Rev Cent Dermatol Pascua. 2020; 29 (2-3): 86-89. https:// dx.doi.org/10.35366/99921 
tosómico dominante. Se desarrolla durante el periodo neonatal, o poco tiempo después. Afecta principalmente a los hombres. La forma adquirida es mucho más frecuente, se observa principalmente en mujeres jóvenes, y puede o no asociarse a las patologías mencionadas previamente. $^{7}$

Clínicamente la TNU se caracteriza por la presencia de múltiples telangiectasias distribuidas de forma lineal, unilateral, siguiendo el trayecto de un dermatoma, o las líneas de Blaschko, con una predilección por el hemicuerpo superior hasta en $80 \%$, afectando principalmente la cara, cuello, hombro, brazo y tórax. ${ }^{7,8}$ En algunos casos puede observarse un halo blanquecino, también llamado halo anémico alrededor de las telangiectasias..$^{7,9}$

Las imágenes histopatológicas muestran cortes constituidos por múltiples vasos de pequeño calibre de paredes delgadas, dilatados, congestionados, ubicados en la dermis superficial, media, y en menor cantidad en la dermis profunda. No se observan datos de neoangiogénesis. ${ }^{1,7,10}$

El diagnóstico se realiza mediante correlación clínicohistopatológica.

Los principales diagnósticos diferenciales que hay que considerar son: el angioma serpiginoso de Hutchinson, la telangiectasia hemorrágica familiar, la telangiectasia esencial generalizada, la telangiectasia macularis eruptiva perstans y los angiomas estelares simples. ${ }^{6,10}$

La TNU suele ser un padecimiento persistente de evolución crónica. No existe un tratamiento específico, aunque los casos adquiridos en algunas ocasiones pueden remitir de manera espontánea si se detecta la causa subyacente.

Por la evolución y naturaleza de esta patología, se sugiere únicamente mantener en observación al paciente. Dentro de las opciones terapéuticas disponibles se encuentran el uso de camuflaje cosmético, la electrocoagulación y el empleo de diversas modalidades de láser como el de luz pulsada, $\mathrm{Nd}$ :YAG y $\mathrm{CO}_{2}$, todos ellos con resultados variables. ${ }^{1,6,9}$

\section{CASO CLÍNICO}

Se trata de una mujer de 33 años de edad, soltera, originaria y residente de la Ciudad de México. En cuanto a antecedentes personales patológicos, únicamente destaca la realización de una cirugía cardiaca a los tres años de edad por probable conducto arterial persistente. En lo relacionado a sus antecedentes ginecoobstétricos, refiere uso de dispositivo intrauterino liberador de levonorgestrel durante los últimos tres años. Resto de antecedentes negativos o irrelevantes para el padecimiento actual. La paciente acude a consulta por una dermatosis diseminada a tronco y extremidad superior izquierda. De la primera compromete cara superior y posterior de hombro, y cara posterior del hemitórax izquierdo en su tercio superior. De la segunda, afecta cara anterolateral del brazo, pliegue antecubital, cara anterolateral del antebrazo y dorso de la mano. Es unilateral. La dermatosis es de aspecto monomorfo, constituida por numerosas manchas vasculares de forma y tamaño variable, bien limitadas, así como algunas otras manchas color café oscuro, y algunas telangiectasias que siguen un trayecto que tiende a ser lineal, discontinuo. De evolución crónica y asintomática (Figuras 1 a 3). Al interrogatorio dirigido, la paciente refiere una evolución de siete meses, con aparición de las primeras lesiones en pliegue antecubital, las cuales posteriormente aumentaron en número, diseminándose al resto de los segmentos corporales ya mencionados. Acudió con un médico particular, quien inició tratamiento con loratadina, cloranfenicol e hidrocortisona en crema por tiempo no especificado, sin mejoría, por lo que suspendió dicho tratamiento y decide acudir a esta unidad médica para valoración y tratamiento.

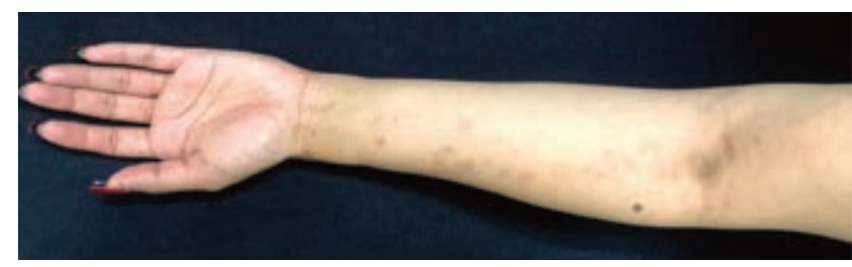

Figura 1: Numerosas manchas color café oscuro, algunas otras de aspecto vascular y telangiectasias en trayecto lineal no continuo en cara anterior del antebrazo.

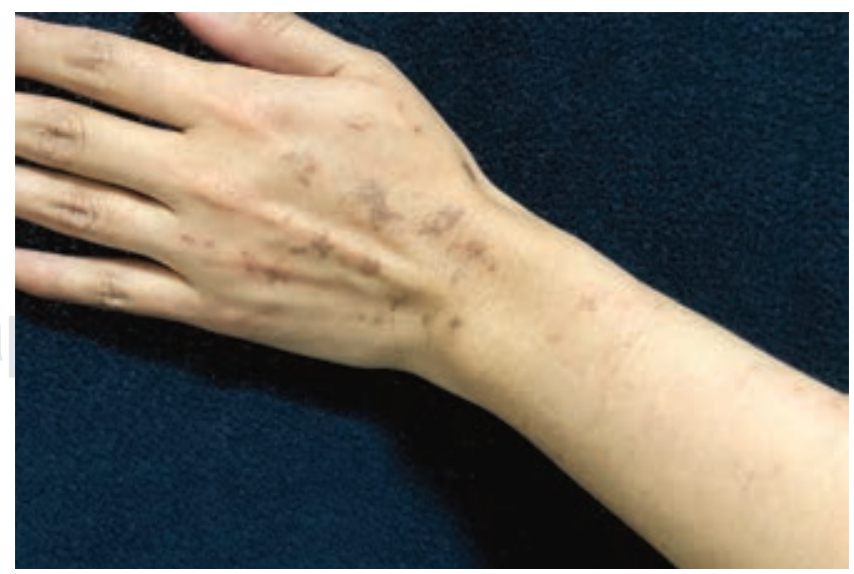

Figura 2: Telangiectasias y manchas oscuras y vasculares en cara posterior de antebrazo. 
Con el diagnóstico clínico presuntivo de telangiectasia nevoide unilateral versus angioma serpiginoso, se realizó una biopsia incisional de una de las lesiones del hombro. El estudio histopatológico describe una epidermis con escasa capa córnea, aplanamiento de los procesos interpapilares e hiperpigmentación de la capa basal. En dermis superficial se observan vasos dilatados y congestionados, rodeados por infiltrados moderadamente densos, constituidos por linfocitos e histiocitos, además de algunas zonas con extravasación de eritrocitos; resto del corte sin alteraciones (Figuras 4 y 5). Con esto se realiza un diagnóstico histopatológico de dermatitis perivascular superficial con extravasación de eritrocitos, y al realizar correlación clínico-histopatológica se concluye que se trata de un caso de telangiectasia nevoide unilateral adquirida (TNUA). Se solicitan estudios de laboratorio para completar el abordaje (biometría hemática, química sanguínea, perfil de hepatitis viral, prueba de embarazo), todos ellos con resultados dentro de parámetros normales. Se explica ampliamente a la paciente la naturaleza y evolución de su padecimiento. Al ser el dispositivo intrauterino liberador de levonorgestrel el único factor hormonal identificado en la paciente, se sugiere que, en conjunto con el Servicio de Ginecología, se considere cambiar el método de planificación familiar por uno no hormonal. Se da tratamiento con medidas generales.

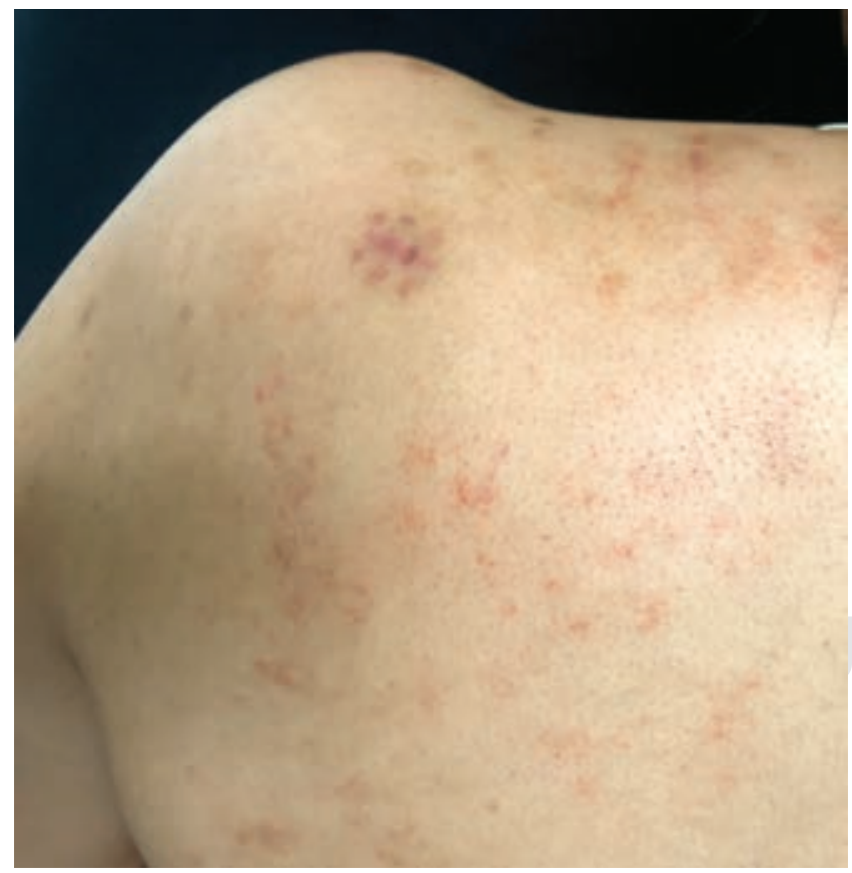

Figura 3: Lesiones vasculares que se extienden desde el hombro y cara posterior del hemitórax izquierdo.

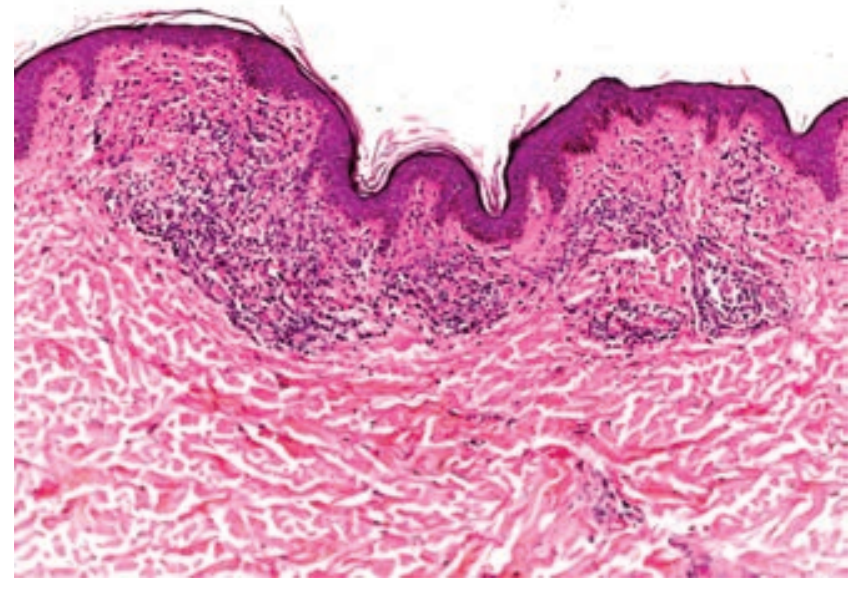

Figura 4: Epidermis con aplanamiento de los procesos interpapilares (H\&E 10x).

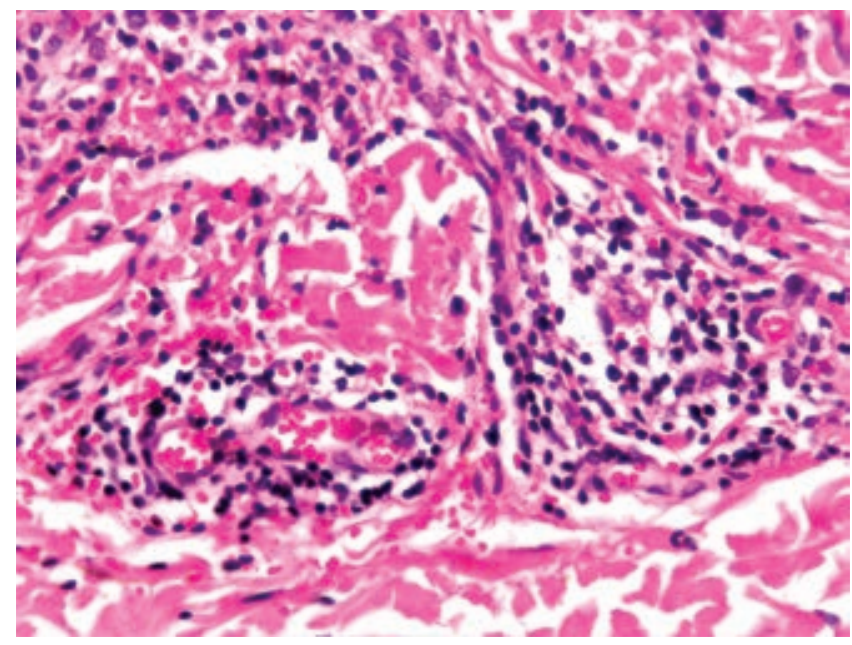

Figura 5: Vasos dilatados y congestionados rodeados por moderados infiltrados linfohistiocitarios (H\&E 40X).

\section{COMENTARIO}

Este caso ejemplifica las manifestaciones de una dermatosis muy poco frecuente, que comparte características clínicas con lo publicado en la literatura internacional. Su diagnóstico es un reto para el médico tratante, considerando su baja frecuencia y grado de sospecha clínica.

Éste es el tercer caso registrado en el Centro Dermatológico Pascua en las últimas dos décadas.

Es importante recordar que, al realizar el abordaje diagnóstico, es imperativo descartar otras patologías y tratar de identificar algún factor desencadenante 
para tratarlo lo más pronto posible. En el caso de la paciente, motivo de esta comunicación, no pudo identificarse ninguno.

Por otro lado, se debe comunicar de forma adecuada al paciente el pronóstico y naturaleza de su dermatosis pues, aunque tiene un curso benigno, el impacto por las alteraciones cosméticas que pueden desarrollarse en estos pacientes es importante, por lo que deben considerarse y ofrecer las opciones terapéuticas pertinentes.

\section{REFERENCIAS}

1. Navarrete FG, González GM, Espinosa AA, Maya ASE, López ZI, Gaxiola AEA. Telangiectasia nevoide unilateral asociada con hepatitis tipo A. Dermatol Rev Mex. 2013; 57: 136-139.

2. Hynes LR, Shenefelt PD. Unilateral nevoid telangiectasia: occurrence in two patients with hepatitis C. J Am Acad Dermatol. 1997; 36: 819-822.

3. Sadick NS, Urmacher C. Estrogen and progesterone receptors: their role in postsclerotherapy angiogenesis telangiectatic matting. Dermatol Surg. 1999; 25: 539-543.

4. Karabudak O, Dogan B, Taskapan O, Harmanyeri Y. Acquired unilateral nevoid telangiectasia syndrome. J Dermatol. 2006; 33: 825-826.

5. Rodríguez-Bandera Al, Feito-Rodríguez M, Vorlicka K, De LucasLaguna R. Líneas de Blaschko y otros mosaicismos cutáneos. Piel. 2013; 28: 457-468.
6. Arias M, González R, Retamar R, López MC, Demarchi M, Kien MC et al. Telangiectasia nevoide unilateral. Comunicación de cuatro casos. Dermatología Argentina Unidad De Dermatología Hospital General de Agudos "Dr. Cosme Argerich". Centro Chouela Dermatología. 2008 15: 44-48.

7. Guedes R, Leite L. Unilateral nevoid telangiectasia: a rare disease? Indian J Dermatol. 2012; 57: 138-140. doi: 10.4103/00195154.94288.

8. Kreft B, Marsch WC, Wohlrab J. Unilateral nevoid telangiectasia syndrome. Dermatology. 2004; 209: 215-217.

9. Ramírez-Andreo A, Gómez-Avivar P, Tercedor J, Fernández-Vilariño E, Morales E, García-López $C$ et al. Telangiectasia nevoide unilateral. Actas Dermosifiliogr. 2003; 94: 258-259.

10. Cardona-Hernández MA, Navarrete FG, González GM, Cabrera PA. Telangiectasia nevoide unilateral. Rev Cent Dermatol Pascua. 2015; 24: $52-55$

Correspondencia:

Dr. Juan Ramón Trejo Acuña

Dr. Vértiz Núm. 464 Esq. Eje 3 Sur,

Col. Buenos Aires, 06780,

Alcaldía Cuauhtémoc, CDMX.

Tel. 5519-6351.

E-mail: hermesjuanderma@hotmail.com 